\title{
Draft Genome Sequence of Kocuria rhizophila strain TPW45, an Actinobacterium Isolated from Freshwater
}

\author{
Tan-Guan-Sheng Adrian, Pui-Wan Tan, Jian-Woon Chen, Wai-Fong Yin, Kok-Gan Chan $\bowtie$ \\ Division of Genetics and Molecular Biology, Institute of Biological Sciences, Faculty of Science, University of Malaya, Kuala Lumpur, \\ Malaysia
}

$\square$ Corresponding author: Division of Genetics and Molecular Biology, Institute of Biological Sciences, Faculty of Science, University of Malaya, Kuala Lumpur 50603, Malaysia; E-mail: kokgan@um.edu.my

(1) Ivyspring International Publisher. Reproduction is permitted for personal, noncommercial use, provided that the article is in whole, unmodified, and properly cited. See http://ivyspring.com/terms for terms and conditions.

Published: 2016.05 .06

\begin{abstract}
Kocuria rhizophila is a ubiquitous bacterium which is well known for its industrial value. Here, we present the draft genome of Kocuria rhizophila strain TPW45 which was isolated from Sungai Gabai, Selangor, Malaysia. The assembled genome comprised of 46 contigs and the estimated genome size is $2.7 \mathrm{Mb}$. Based on the RAST annotation, a gene cluster responsible for aromatic compound degradation was identified in this strain.
\end{abstract}

Key words: Kocuria rhizophila, Phenylacetate degradation, Whole genome sequencing

\section{Introduction}

Kocuria rhizophila is a Gram-positive, coccoid, and spherical bacterium belonging to the family Micrococcaceae in the phylum Actinobacteria, and was designated as a new species by Kovács and coworkers (1). Members of the genus Kocuria have been isolated from a wide variety of natural sources, and $K$. rhizophila in particular, was found to have important industrial applications. K. rhizophila has been utilised as the standard quality control strain for antimicrobial susceptibility testing (2) and also as a decolorizing agent of industrial dyes (3). Our strain was isolated from Sungai Gabai, Selangor, Malaysia and analysis of the genome revealed the presence of an aromatic compound degradation pathway.

Initially, the genomic DNA of $K$. rhizophila TPW45 was extracted using MasterPure ${ }^{\mathrm{TM}}$ Complete DNA and RNA Purification Kit (Epicentre, Inc., Madison, WI, USA) according to the manufacturer's recommended protocol. Subsequently, the quality of DNA was examined using the NanoDrop 2000 spectrophotometer (Thermo Scientific) and quantified using Qubit 2.0 fluorometer (Life Technologies). For whole-genome sequencing of $K$. rhizophila TPW45, the library was prepared using Illumina Nextera DNA Sample Preparation Kit as per manufacturer's guidelines. The sequencing of this strain was then performed on the Illumina HiSeq 2500 (Illumina, Inc., CA) using a paired-end library with an average insert size of $702 \mathrm{bp}$, obtaining 3,407,419 raw reads of $100 \mathrm{bp}$ in length. After trimming, de novo assembly of the genome was performed using CLC Genomics Workbench 7.0 (4) while the genome annotation was performed using Rapid Annotation using Subsystem Technology (RAST) (5).

The assembled genome consists of 2,701,701 bases across 46 contigs with an $\mathrm{N}_{50}$ contig length of 114,097 bases. The average genome coverage was found to be $202.76 \times$. The assembled genome was found to have a $\mathrm{G}+\mathrm{C}$ content of $70.6 \%$, falling within the range of known K. rhizophila genomes (Table 1). There were 2,349 predicted coding DNA sequences (CDS), whereas a total of 50 tRNAs were predicted using tRNAscan-SE (6), and 4 rRNAs was identified by using RNAmmer (7). This draft genome revealed a 
putative gene cluster involved in aromatic compound degradation that encodes a phenylacetatic acid (PAA) pathway. This PAA gene cluster was found in contig 17 of our strain and the gene arrangement was found to be similar to that of K. rhizophila strain DC2201, which is a closely related strain (Figure 1). Phenylacetate or phenylacetyl-coenzyme A (CoA) can be found as a derivative product from many substrates namely phenylalanine, 2-phenylethylamine, some phenylalkanoic acids, and also pollutants such as styrene and ethylbenzene. (8) The PAA pathway proceeds via phenylacetyl-coA and ring fission is hydrolysed via a multicomponent oxygenase encoded by paaGHIJK. (9)

In summary, phenylacetate degradation has been more widely studied in Proteobacteria such as Escherichia spp. and Pseudomonas spp. compared to Gram-positive bacteria. (8) We hope that this genome sequence of strain TPW45 will provide more insight into the phenylacetate degradation pathway by $K$. rhizophila. Further analysis of this genome and supporting experimental evidence will be needed to support this prediction and further understand the process of aromatic compound degradation by bacteria via PAA catabolism.

Table 1 Genome features of Kocuria rhizophila strain TPW45 and K. rhizophila reference sequences from RAST.

\begin{tabular}{llllllll}
\hline Strain & Source & $\begin{array}{l}\text { Size } \\
(\mathrm{Mb})\end{array}$ & $\begin{array}{l}\text { No. of } \\
\text { Contig } \\
(\mathrm{s})\end{array}$ & $\begin{array}{l}\mathrm{G}+\mathrm{C}) \\
\text { (\%) }\end{array}$ & $\begin{array}{l}\text { No. of } \\
\text { CDS }\end{array}$ & $\begin{array}{l}\text { No. of } \\
\text { rRNA }\end{array}$ & $\begin{array}{l}\text { No. of } \\
\text { tRNA }\end{array}$ \\
\hline PC2201 & Soil & 2.70 & 1 & 71.2 & 2,357 & 3 & 46 \\
& $\begin{array}{l}\text { Intestine of } \\
\text { rabbitfish } \\
\text { (Siganus } \\
\text { doliatus) }\end{array}$ & 2.82 & 75 & 70.4 & 2,370 & 5 & 46 \\
TPW45 & $\begin{array}{l}\text { This study } \\
\text { 14ASP }\end{array}$ & 2.70 & 46 & 70.6 & 2,349 & 4 & 50 \\
& $\begin{array}{l}\text { Endophyte } \\
\text { of Oxalis } \\
\text { corniculata }\end{array}$ & 2.70 & 183 & 70.8 & 1,900 & 8 & 46 \\
\hline
\end{tabular}

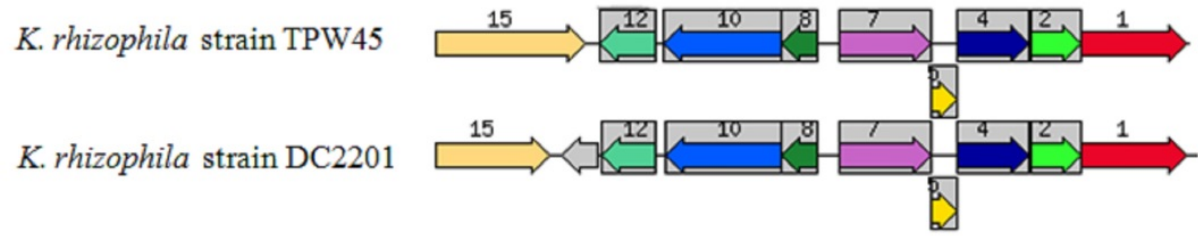

Figure 1 Orientation and annotation of phenylacetic acid (PAA) gene cluster involved in K. rhizophila strain TPW45 and DC2201 as identified from RAST server. (1) Phenylacetate-CoA oxygenase/reductase, PaaK subunit; (2) Phenylacetate-CoA oxygenase, Paaj subunit; (4) Phenylacetate-CoA oxygenase, Paal subunit; (5) Phenylacetate-CoA oxygenase, PaaH subunit; (7) Phenylacetate-CoA oxygenase, PaaG subunit; (8) Phenylacetic acid degradation protein PaaD, thioesterase; (10) Phenylacetate-coenzyme A ligase, PaaF; (12) Transcriptional regulator, TetR family; (15) Aldehyde dehydrogenase , PaaZ

\section{Nucleotide sequence accession number}

The whole genome shotgun project has been deposited in DDBJ/EMBL/GenBank under accession number JWTC00000000.1.

\section{Acknowledgments}

This work was supported by the University of Malaya for High Impact Research Grant (UM-MOHE HIR Nature Microbiome Grant No. H-50001-A000027) awarded to Kok-Gan Chan.

\section{Competing Interests}

The authors have declared that no competing interest exists.

\section{References}

1. Kovács G, Burghardt J, Pradella S, et al. Kocuria palustris sp. nov. and Kocuria rhizophila sp. nov., isolated from the rhizoplane of the narrow-leaved cattail (Typha angustifolia). Int J Syst Bacteriol. 1999; 49: 167-173.

2. Takarada $\mathrm{H}$, Sekine $\mathrm{M}$, Kosugi $\mathrm{H}$, et al. Complete genome sequence of the soil actinomycete Kocuria rhizophila. J Bacteriol. 2008; 190(12): 4139-4146.

3. Parshetti G, Kalme S, Saratale G, et al. Biodegradation of malachite green by Kocuria rosea MTCC 1532. Acta Chim Slov. 2006; 53(4): 492.

4. [Internet] CLC Genomics Workbench. https://www.clcbio.com

5. Aziz RK, Bartels D, Best AA, et al. (2008). The RAST Server: rapid annotations using subsystems technology. BMC Genomics. 2008; 9: 75.

6. Schattner P, Brooks AN, Lowe TM. The tRNAscan-SE, snoscan and snoGPS web servers for the detection of tRNAs and snoRNAs. Nucleic Acids Res. 2005; 33 (Web server issue): W686-W689.

7. Lagesen K, Hallin P, Rødland EA, et al. RNAmmer: consistent and rapid annotation of ribosomal RNA genes. Nucleic Acids Res. 2007; 35(9): 3100-3108. 
8. Teufel $\mathrm{R}$, Mascaraque $\mathrm{V}$, Ismail $\mathrm{W}$, et al. Bacterial phenylalanine and phenylacetate catabolic pathway revealed. Proceedings of the National Academy of Sciences. 2010, 107(32): 14390-14395.

9. Alvarez HM. Biology of Rhodococcus. Berlin, Germany: Springer-Verlag Berlin Heidelberg; 2010. 